\title{
Predictors of infection after 754 cranioplasty operations and the value of intraoperative cultures for cryopreserved bone flaps
}

\author{
Ryan P. Morton, MD, I. Josh Abecassis, MD, ${ }^{1}$ Josiah F. Hanson, BA, ${ }^{1}$ Jason Barber, MS, ${ }^{1}$ \\ John D. Nerva, MD, ${ }^{1}$ Samuel N. Emerson, MD, PhD, ${ }^{1}$ Chibawanye I. Ene, MD, PhD, ${ }^{1}$ \\ Michelle M. Chowdhary, MD, ${ }^{1}$ Michael R. Levitt, MD, ${ }^{1}$ Andrew L. Ko, MD, ${ }^{1}$ Timothy H. Dellit, MD, ${ }^{2}$ \\ and Randall M. Chesnut, MD'1
}

Departments of ${ }^{1}$ Neurological Surgery and ${ }^{2}$ Infection Control, Harborview Medical Center, University of Washington School of Medicine, Seattle, Washington

OBJECTIVE The authors' aim was to report the largest study on predictors of infection after cranioplasty and to assess the predictive value of intraoperative bone flap cultures before cryopreservation.

METHODS They retrospectively examined all cranioplasties performed between March 2004 and November 2014. Throughout this study period, the standard protocol during initial craniectomy was to obtain a culture swab of the extracted autologous bone flap (ABF) - prior to its placement in cytostorage-to screen for microbial contamination. Two consecutive protocols were employed for the use and interpretation of the intraoperative swab culture results: A) From March 2004 through June 2013, any culture-positive ABF (+ABF) was discarded and a custom synthetic prosthesis was implanted at the time of cranioplasty. B) From July 2013 through November 2014, any ABF with a skin flora organism was not discarded. Instead, cryopreservation was maintained and the +ABF was re-implanted after a 10-minute soak in bacitracin irrigation as well as a 3-minute soak in betadine.

RESULTS Over the 10.75 -year period, 754 cranioplasty procedures were performed. The median time from craniectomy to cranioplasty was 123 days. Median follow-up after cranioplasty was 237 days for protocol $A$ and 225 days for protocol B. The overall infection rate after cranioplasty was $6.6 \%$ (50 cases) occurring at a median postoperative Day 31 . Staphylococcus spp. were involved as the causative organisms in $60 \%$ of cases.

Culture swabs taken at the time of initial craniectomy were available for $640 \mathrm{ABFs}$ as $114 \mathrm{ABFs}$ were not salvageable. One hundred twenty-six (20\%) were culture positive. Eighty-nine +ABFs occurred during protocol $A$ and were discarded in favor of a synthetic prosthesis at the time of cranioplasty, whereas $37+A B F s$ occurred under protocol $B$ and were reimplanted at the time of cranioplasty.

Cranioplasty material did not affect the postcranioplasty infection rate. There was no significant difference in the infection rate among sterile $A B F s(7 \%),+A B F s(8 \%)$, and synthetic prostheses $(5.5 \% ; p=0.425)$. All $3+A B F$ infections under protocol $B$ were caused by organisms that differed from those in the original intraoperative bone culture from the initial craniectomy. A cranioplasty procedure $\leq 14$ days after initial craniectomy was the only significant predictor of postcranioplasty infection ( $p=0.007, \mathrm{HR} 3.62)$.

CONCLUSIONS Cranioplasty procedures should be performed at least 14 days after initial craniectomy to minimize infection risk. Obtaining intraoperative bone cultures at the time of craniectomy in the absence of clinical infection should be discontinued as the culture results were not a useful predictor of postcranioplasty infection and led to the unnecessary use of synthetic prostheses and increased health care costs.

http://thejns.org/doi/abs/10.3171/2015.8.JNS151390

KEY WORDS cranioplasty; infection; cryopreserved; intraoperative cultures; PEEK; diagnostic and operative techniques; polyetheretherketone

ABBREVIATIONS ABF = autologous bone flap; $+\mathrm{ABF}=$ culture-positive $\mathrm{ABF} ; \mathrm{PEEK}=$ polyetheretherketone; $\mathrm{TBI}$ = traumatic brain injury.

SUBMITTED June 15, 2015. ACCEPTED August 13, 2015.

INCLUDE WHEN CITING Published online January 15, 2016; DOI: 10.3171/2015.8.JNS151390. 
$\mathrm{C}$ RANIOPLASTY aims to restore a cosmetic skull defect with either a stored autologous bone flap (ABF) or a custom synthetic prosthesis. This relatively common neurosurgical procedure is associated with complication rates of $15 \%-35 \%, 4,6,8-10,12-20,22-29$ with the most frequent complication being postoperative infection. The highest reported clinical infection rate after cranioplasty is $26 \% .{ }^{29}$ As native skin flora (for example, Staphylococcus and Propionibacterium) represent the most common source of postoperative infection, ${ }^{3,29}$ our institutionalong with others ${ }^{5}$ - has established a protocol of acquiring intraoperative culture swabs of the removed bone flap at the time of initial craniectomy to screen for microbial contamination before cryopreservation and eventual reimplantation. The potential clinical benefit of this methodology is unknown. ${ }^{2}$ We reviewed our experience at a highvolume tertiary care referral center over the past 10 years to present the largest single-center study on cranioplasty infection to date, with the goal of determining predictors of cranioplasty infections and the clinical utility of intraoperative bone flap cultures.

\section{Methods}

The hospital's institutional review board approved this study. We conducted an electronic search of our institution's operative reports and retrospectively examined all cranioplasties performed from March 2004 through November 2014. Each case was then individually reviewed to ensure accuracy. Clinical and radiographic data were recorded. Per protocol at our institution, any post-cranioplasty patient with signs of infection was routinely treated by immediate explantation of the ABF followed by the administration of culture-tailored antibiotics for 6-12 weeks as guided by the infectious disease team. Thus, "postcranioplasty infection," for the purposes of this study, is defined as an infection resulting in removal of the ABF.

\section{Intraoperative Bone Swab}

Throughout the study period, our standard institutional protocol during the initial craniectomy was to obtain a culture swab of the extracted ABF prior to its placement in cytostorage for the future cranioplasty procedure. If the $\mathrm{ABF}$ was unsalvageable (for example, a fragmented, open fracture with environmental contamination, invasion by tumor, and so forth), a swab was not obtained and the ABF was not cryopreserved. Cultures were monitored during the patient's recovery to screen for microbial contamination of the ABF. During the study period, we employed 2 consecutive protocols for the use and interpretation of the intraoperative swab culture results: A) From March 2004 through June 2013, any culture-positive ABF (+ABF), regardless of organism, was discarded and a custom synthetic prosthesis was implanted at the time of cranioplasty. B) From July 2013 through November 2014, any ABF with a skin flora organism was not discarded. Instead, cryopreservation was maintained after the $+\mathrm{ABF}$ was soaked in bacitracin $(50,000 \mathrm{U}$ in $500 \mathrm{ml}$ saline) for 10 minutes prior to freezing. During thawing and prior to reimplantation, the ABF was again soaked in bacitracin for at least 10 minutes and subjected to a 3-minute soak in betadine solution as well.

\section{Statistical Analysis}

Statistical analysis was performed using SPSS version 19 (IBM Corp.). Cox proportional-hazards regression models were used to analyze variables potentially influencing the risk of infection. A p value $<0.01$ was considered significant.

\section{Results}

Over the 10.75-year period, 754 cranioplasty procedures were performed. Four-hundred fifty-five patients were male $(60 \%)$, and the average patient age was 44 years (range 1-86 years). Six hundred forty patients were managed under protocol A and 114 patients under protocol B. Indications for craniectomy are shown in Table 1, with traumatic brain injury (TBI) as the leading indication in $51.4 \%$ (388) of cases. The median time from craniectomy to cranioplasty was 123 days (range 2-9855 days). Median follow-up after cranioplasty was 237 days for protocol A (range 7 days-10 years) and 225 days for protocol B (range 30-480 days).

\section{Material Used for Cranioplasty}

A sterile ABF was used in 532 cases (70.6\%). A synthetic flap was used in 222 cases (29.4\%). Eighty-nine +ABFs were discarded during the time of protocol $\mathrm{A}$ in favor of a synthetic flap, but $37+$ ABFs were reimplanted during the period of protocol B. The material used for the synthetic prostheses subgroup varied (Table 2), with polyetheretherketone (PEEK) as the most common. The indication for a synthetic prosthesis varied: $71(32 \%)$ for an initial unsalvageable ABF, 39 (17.6\%) for an ABF removed after intracranial infection (for example, brain abscess, elective craniotomy infection), $4(1.8 \%)$ due to tumor invasion of the $\mathrm{ABF}$, and $19(8.5 \%)$ at the discretion of the treating physician. However, the leading indication for implantation of a synthetic flap was a culture-positive bone swab $(+\mathrm{ABF})$ from the original craniectomy in the absence of clinical suspicion for infection in 89 cases (40.1\%) during the study period under protocol A.

\section{Postcranioplasty Infection}

The overall infection rate after cranioplasty was $6.6 \%$ (50 cases) occurring at a median postoperative Day (POD) 31 (range 8-2035 days). Eleven cases (22\%) occurred after POD 90. Causative organisms are listed in Table 3. Isolated Gram-positive infections were the most common $(58 \%)$, whereas isolated Gram-negative infections were cultured in $18 \%$ of cases and polymicrobial infections in $16 \%$ of cases. Staphylococcus spp. were the causative organisms in $62 \%$ of cases.

Cranioplasty material did not affect the postcranioplasty infection rate, as there was no significant difference among sterile ABFs (7\%), +ABFs (8\%), and synthetic prostheses $(5.5 \%$; $\mathrm{p}=0.425$; Table 2$)$. A cranioplasty procedure $\leq 14$ days after the initial craniectomy was the only significant predictor of postcranioplasty infection $(\mathrm{p}=0.007$, HR 3.62). The following factors were evaluated but were found to not statistically influence postoperative infection: age; sex; indication for original craniectomy; cranioplasty performed at $\leq 30$, $\leq 90$, or $>90$ days after craniectomy; shunt placement during cranioplasty; postcranioplasty 
TABLE 1. Indication for craniectomy in a population of 754 cranioplasty patients

\begin{tabular}{lr}
\hline \multicolumn{1}{c}{ Indication } & No. (\%) \\
\hline $\begin{array}{l}\text { TBI (depressed skull fracture, extraaxial hematoma, intra- } \\
\text { axial hematoma, \&/or diffuse swelling) }\end{array}$ & $388(51)$ \\
\hline Ischemic stroke w/ malignant edema & $73(10)$ \\
\hline Intraparenchymal hemorrhage (spontaneous or AVM) & $104(14)$ \\
\hline Subarachnoid hemorrhage & $102(14)$ \\
\hline Spontaneous brain abscess & $5(1)$ \\
\hline Tumor & $22(3)$ \\
\hline Postop infection of a previous craniotomy & $39(5)$ \\
\hline Other & $21(3)$ \\
\hline
\end{tabular}

$\mathrm{AVM}=$ arteriovenous malformation.

hydrocephalus requiring a separate shunting procedure; postoperative seizures; postoperative hematoma requiring evacuation; and place of discharge after cranioplasty. Neither did the type of synthetic material used influence the postoperative infection rates within the synthetic cranioplasty subgroup ( $\mathrm{p}=0.986$; Table 2$)$.

\section{Intraoperative Bone Culture}

Culture swabs taken at the time of initial craniectomy were available for 640 subsequent cranioplasties. One hundred fourteen ABFs were not salvageable due to gunshot wound, open depressed skull fracture, infection of prior craniotomy, initial craniectomy at an outside facility, and so forth. One hundred twenty-six ABFs (20\%) were culture positive $(+\mathrm{ABF})$. Eighty-nine $+\mathrm{ABFs}$ occurred during protocol A and were discarded, and a synthetic prosthesis was used at cranioplasty. Thirty-seven $+\mathrm{ABFs}$ occurred under protocol B and were replaced at the time of cranioplasty after a bacitracin and betadine soak.

The 37 +ABFs reimplanted under protocol B had cultures positive for Propionibacterium acnes (22 cases), coagulase-negative Staphylococcus aureus (9 cases), Propionibacterium acnes and coagulase-negative Staphylococcus aureus (2 cases), Micrococcus (1 case), Diphtheroids spp. (1 case), Candida parapsilosis (1 case), and Bacillus spp. (1 case). There were 3 postcranioplasty infections (8\%) after $+\mathrm{ABF}$ operations under protocol $\mathrm{B}$, a rate similar to the overall infection rate for all cranioplasty proce-
TABLE 3. Causative organisms in 50 cranioplasty infections

\begin{tabular}{|c|c|}
\hline Organism & No. \\
\hline \multicolumn{2}{|l|}{ Isolated single Gram-positive infection $(n=29)$} \\
\hline MRSA & 12 \\
\hline MSSA & 11 \\
\hline Propionibacterium & 4 \\
\hline Staphylococcus epidermidis & 1 \\
\hline Group A strep & 1 \\
\hline \multicolumn{2}{|l|}{ Isolated single Gram-negative infection $(n=9)$} \\
\hline Morganella morganii & 1 \\
\hline Serratia marcescens & 2 \\
\hline Enterobacter & 2 \\
\hline Escherichia coli & 3 \\
\hline Pseudomonas & 1 \\
\hline \multicolumn{2}{|l|}{ Polymicrobial infection $(n=8)$} \\
\hline MSSA, Fusobacterium nucleatus & 1 \\
\hline CoNS, Streptococcus milleri, Candida albicans & 1 \\
\hline MRSA, MSSA, Propionibacterium & 1 \\
\hline MRSA, Proteus mirabilis & 1 \\
\hline CoNS, MSSA & 1 \\
\hline MSSA, Candida & 1 \\
\hline $\begin{array}{l}\text { Propionibacterium, Finegoldia magna, Enterobacter } \\
\text { aerogenes }\end{array}$ & 1 \\
\hline Propionibacterium, MSSA & 1 \\
\hline No culture growth & 2 \\
\hline Cranioplasty removal at outside hospital & 2 \\
\hline
\end{tabular}

CoNS = coagulase-negative Staphylococcus aureus; $\mathrm{MRSA}=$ methicillin resistant $S$. aureus; MSSA = methicillin-sensitive $S$. aureus.

dures (Table 2). All $3+\mathrm{ABF}$ infections under protocol B were caused by organisms that were different from those in the original intraoperative bone culture from the initial craniectomy.

\section{Discussion}

Cranioplasty is a common neurosurgical procedure that, given the implantation of nonliving tissue (whether $\mathrm{ABF}$ or synthetic prosthesis), carries a risk of complica-

TABLE 2. Material used for 754 cranioplasties and the rate of infection

\begin{tabular}{lcccc}
\hline Cranioplasty Material $^{*}$ & No. $(\%)$ & No. of Observed Infections (\%) & Hazard Ratio & $95 \% \mathrm{Cl}$ \\
\hline Sterile ABF & $495(66)$ & $35(7)$ & {$[1.00] \dagger$} & \\
\hline+ ABF & $37(5)$ & $3(8)$ & 1.33 & $(0.41,4.34)$ \\
\hline Synthetic material $\ddagger$ & $222(29)$ & $12(5)$ & 0.67 & $(0.34,1.33)$ \\
\hline PEEK & $151(68)$ & $9(6)$ & {$[1.00] \dagger$} & \\
\hline Porex & $24(11)$ & $1(4)$ & 0.77 & $(0.10,6.12)$ \\
\hline Methylmethacrylate only & $7(3)$ & $0(0)$ & 0.00 & \\
\hline MedPor & $23(10)$ & $2(9)$ & 1.48 & $(0.31,7.00)$ \\
\hline Other & $17(8)$ & $0(0)$ & 0.00 & \\
\hline
\end{tabular}

MedPor = porous polyethylene; Porex $=$ porous high-density polyethylene .

* $p=0.425$.

$\dagger$ Reference.

$\ddagger p=0.986$. 
tions such as surgical site infection. The use of intraoperative bone culture at the time of craniectomy is thought to indicate whether the initial ABF is sterile for future reimplantation. After reviewing our large series of cranioplasty procedures, we found no significant association between $+\mathrm{ABF}$ and postcranioplasty infection. The overall infection rate of $6.6 \%$ is slightly lower than the $7.9 \%$ rate reported in a recent systematic review. ${ }^{28}$ Notably, $22 \%$ of the postcranioplasty infections in the present study occurred more than 90 days after the index procedure and would not be considered surgical site infections under the guidelines of the Centers for Disease Control and Prevention's National Healthcare Safety Network.

Ultra-early cranioplasty ( $\leq 14$ days after initial craniectomy) was the only patient or procedural variable associated with postcranioplasty infection. Importantly, it is one of the few variables over which the treating physician has control. Previous studies have reported conflicting results regarding the impact of the timing of cranioplasty on infection rates. ${ }^{4,15,20,22,24,25,29}$ A large systematic review ${ }^{28}$ failed to show any statistically significant influence on infection when comparing "early" ( $<3$ months) and late cranioplasty. However, small sample sizes limited the statistical power of that review, and the time to cranioplasty evaluated was only 3 months. The influence of ultra-early ( $\leq 14$ days) cranioplasty has not been evaluated in the literature.

In our series, ultra-early cranioplasty was performed during the initial hospitalization for craniectomy, after the resolution of cerebral edema. In these cases, there was a logistical desire by both the physician and the patient's family to replace the ABF during the primary hospitalization rather than after discharge. The total number of patients (27) who underwent an ultra-early cranioplasty was relatively small, but the infection rate of $18.5 \%$ among this group was significant and probably represents an avoidable risk. Replacing the bone flap 30 days later, which would still be considered early according to several previous studies, did not confer additional infection risk. Such timing is favored by some centers given reports of functional improvement after early cranioplasty., ${ }^{1,11,21}$ Our data suggest that the ideal time for early cranioplasty, if desired, is probably between 14 and 30 days to balance the risk of infection with neurocognitive improvement since interrupting the wound healing process earlier than 14 days seems to predispose to subsequent wound complications.

In cases of synthetic prostheses, the choice of material did not impact the risk of infection in our series. A recent international, multiinstitutional report of 66 PEEK prostheses demonstrated an infection rate of 7.6\%. ${ }^{21}$ The postcranioplasty infection rate among 151 PEEK implants in our series was $6 \%$, and, as in other studies, suggests that synthetic prostheses have infection rates similar to those with ABFs. ${ }^{4,14,19,23,25,27-29}$

Most important in our results is the favorable comparison between protocols A and B as regards the reimplantation of $+\mathrm{ABFs}$ despite intraoperative bone cultures of skin flora. Specifically, there was no increased risk of postcranioplasty infection in cases in which a $+\mathrm{ABF}$ was reimplanted, and there was no concordance between the organisms from the few postcranioplasty infections using
$+\mathrm{ABF}$ and the original intraoperative cultured organisms at the time of craniectomy. Another smaller study ${ }^{5}$ also reported no increased risk of infection from the reimplantation of $+\mathrm{ABFs}$ and no agreement between the intraoperative cultures at the time of craniectomy and the causative organism of the postcranioplasty infection.

These results have significantly changed our institutional practice. During the 9.25 years in the study period under protocol A, 89 synthetic prostheses (the majority PEEK) were implanted instead of $+\mathrm{ABFs}$. As the average cost of a PEEK synthetic prosthesis ranges from $\$ 7550$ to $\$ 8350$ depending on its size, had these $+\mathrm{ABF}$ been reimplanted instead (as was done under protocol B) our center would have saved between $\$ 671,950$ and $\$ 743,150$, or up to $\$ 80,340$ annually. We have since permanently abandoned the practice of routine bone cultures at the time of craniectomy and now adhere to protocol B for all bone flaps in the absence of clinical infection.

This study has several limitations. First, while it is the largest cranioplasty series to date, it is still a single-center study primarily composed of patients with TBI and vascular malformation. Additionally, its retrospective nature obviates a proper control group, though the comparison between patients under protocol A and those under protocol B served as a comparison between the 2 reimplantation strategies. Lastly, we defined postcranioplasty infection as an infection requiring cranioplasty explantation. It is possible that patients with minor or superficial infections were not included in our analysis, though the practice of treating such infections with antibiotics and not just explanting the flap is not routine at our institution and probably has a negligible impact on our results. Finally, our protocol B reimplantation strategy included soaking the flap in betadine. While the efficacy of this step is untested and the possibility of untoward influences (such as on reabsorption) is unknown, we cannot generalize our results to the reimplantation of $+\mathrm{ABF}$ without such treatment.

\section{Conclusions}

Intraoperative bone culture at the time of craniectomy is not a clinically useful predictor of postcranioplasty infection, and its elimination may reduce the substantial cost of unnecessary synthetic prostheses. Cranioplasty procedures should be performed at least 14 days after the initial craniectomy to minimize infection risk.

\section{References}

1. Bender A, Heulin S, Röhrer S, Mehrkens JH, Heidecke V, Straube A, et al: Early cranioplasty may improve outcome in neurological patients with decompressive craniectomy. Brain Inj 27:1073-1079, 2013

2. Bhaskar IPIT, Inglis TJ, Bowman J, Lee GY: Microbial contamination assessment of cryostored autogenous cranial bone flaps: should bone biopsies or swabs be performed? Acta Neurochir (Wien) 155:367-371, 2013

3. Bruce JNBS, Bruce SS: Preservation of bone flaps in patients with postcraniotomy infections. J Neurosurg 98:1203-1207, 2003

4. Chang V, Hartzfeld P, Langlois M, Mahmood A, Seyfried D: Outcomes of cranial repair after craniectomy. J Neurosurg 112:1120-1124, 2010

5. Chiang HYSV, Steelman VM, Pottinger JM, Schlueter AJ, 
Diekema DJ, Greenlee JD, et al: Clinical significance of positive cranial bone flap cultures and associated risk of surgical site infection after craniotomies or craniectomies. J Neurosurg 114:1746-1754, 2011

6. Chibbaro S, Di Rocco F, Mirone G, Fricia M, Makiese O, Di Emidio P, et al: Decompressive craniectomy and early cranioplasty for the management of severe head injury: a prospective multicenter study on 147 patients. World Neurosurg 75:558-562, 2011

7. Cong Z SX, Zhang L, Zhao D, Zhou X, Yi C, Shao Y: Early cranioplasty improved rehabilitation in patients with traumatic skull injuries. Neurosurg Q [epub ahead of print], 2014

8. De Bonis P, Frassanito P, Mangiola A, Nucci CG, Anile C, Pompucci A: Cranial repair: how complicated is filling a "hole"? J Neurotrauma 29:1071-1076, 2012

9. Gooch MRGG, Gin GE, Kenning TJ, German JW: Complications of cranioplasty following decompressive craniectomy: analysis of 62 cases. Neurosurg Focus 26(6):E9, 2009

10. Grossman N, Shemesh-Jan HS, Merkin V, Gideon M, Cohen A: Deep-freeze preservation of cranial bones for future cranioplasty: nine years of experience in Soroka University Medical Center. Cell Tissue Bank 8:243-246, 2007

11. Honeybul S, Janzen C, Kruger K, Ho KM: The impact of cranioplasty on neurological function. Br J Neurosurg 27:636-641, 2013

12. Iwama T, Yamada J, Imai S, Shinoda J, Funakoshi T, Sakai $\mathrm{N}$ : The use of frozen autogenous bone flaps in delayed cranioplasty revisited. Neurosurgery 52:591-596, 2003

13. Jho DHNS, Neckrysh S, Hardman J, Charbel FT, AminHanjani S: Ethylene oxide gas sterilization: a simple technique for storing explanted skull bone. Technical note. J Neurosurg 107:440-445, 2007

14. Kriegel RJSC, Schaller C, Clusmann H: Cranioplasty for large skull defects with PMMA (polymethylmethacrylate) or Tutoplast processed autogenic bone grafts. Zentralbl Neurochir 68:182-189, 2007

15. Liang W, Xiaofeng Y, Weiguo L, Gang S, Xuesheng Z, Fei $\mathrm{C}$, et al: Cranioplasty of large cranial defect at an early stage after decompressive craniectomy performed for severe head trauma. J Craniofac Surg 18:526-532, 2007

16. Liao CCKM, Kao MC: Cranioplasty for patients with severe depressed skull bone defect after cerebrospinal fluid shunting. J Clin Neurosci 9:553-555, 2002

17. Lu Y, Hui G, Liu F, Wang Z, Tang Y, Gao S: Survival and regeneration of deep-freeze preserved autologous cranial bones after cranioplasty. Br J Neurosurg 26:216-221, 2012

18. Morina A, Kelmendi F, Morina Q, Dragusha S, Ahmeti F, Morina D, et al: Cranioplasty with subcutaneously preserved autologous bone grafts in abdominal wall-Experience with 75 cases in a post-war country Kosova. Surg Neurol Int 2:72, 2011

19. Movassaghi K, Ver Halen J, Ganchi P, Amin-Hanjani S, Mesa J, Yaremchuk MJ: Cranioplasty with subcutaneously preserved autologous bone grafts. Plast Reconstr Surg 117:202-206, 2006

20. Piedra MPTE, Thompson EM, Selden NR, Ragel BT, Guillaume DJ: Optimal timing of autologous cranioplasty after decompressive craniectomy in children. J Neurosurg Pediatr 10:268-272, 2012
21. Rosenthal G, Ng I, Moscovici S, Lee KK, Lay T, Martin $\mathrm{C}$, et al: Polyetheretherketone implants for the repair of large cranial defects: a 3-center experience. Neurosurgery 75:523-529, 2014

22. Sobani ZASM, Shamim MS, Zafar SN, Qadeer M, Bilal N, Murtaza SG, et al: Cranioplasty after decompressive craniectomy: An institutional audit and analysis of factors related to complications. Surg Neurol Int 2:123, 2011

23. Staffa G, Nataloni A, Compagnone C, Servadei F: Custom made cranioplasty prostheses in porous hydroxy-apatite using 3D design techniques: 7 years experience in 25 patients. Acta Neurochir (Wien) 149:161-170, 2007

24. Stephens FLMC, Mossop CM, Bell RS, Tigno T Jr, Rosner MK, Kumar A, et al: Cranioplasty complications following wartime decompressive craniectomy. Neurosurg Focus 28(5):E3, 2010

25. Walcott BPKC, Kwon CS, Sheth SA, Fehnel CR, Koffie RM, Asaad WF, et al: Predictors of cranioplasty complications in stroke and trauma patients. J Neurosurg 118:757-762, 2013

26. Wiggins A, Austerberry R, Morrison D, Ho KM, Honeybul $\mathrm{S}$ : Cranioplasty with custom-made titanium plates -14 years experience. Neurosurgery 72:248-256, 2013

27. Wurm G, Tomancok B, Holl K, Trenkler J: Prospective study on cranioplasty with individual carbon fiber reinforced polymer (CFRP) implants produced by means of stereolithography. Surg Neurol 62:510-521, 2004

28. Yadla S, Campbell PG, Chitale R, Maltenfort MG, Jabbour $\mathrm{P}$, Sharan AD: Effect of early surgery, material, and method of flap preservation on cranioplasty infections: a systematic review. Neurosurgery 68:1124-1130, 2011

29. Zanaty M, Chalouhi N, Starke RM, Clark SW, Bovenzi CD, Saigh M, et al: Complications following cranioplasty: incidence and predictors in 348 cases. J Neurosurg 123:182188,2015

\section{Disclosures}

Dr. Chesnut is a consultant for Orsan, Neuroptics, and Innerspace.

\section{Author Contributions}

Conception and design: Morton, Abecassis, Nerva, Emerson, Ene, Chowdhary, Levitt, Ko, Dellit, Chesnut. Acquisition of data: Morton, Abecassis, Hanson, Nerva, Emerson, Ene, Chowdhary, Levitt, Dellit. Analysis and interpretation of data: all authors. Drafting the article: Morton, Abecassis, Hanson, Barber, Nerva, Emerson, Ene, Chowdhary, Levitt, Ko, Chesnut. Critically revising the article: Morton, Abecassis, Barber, Chowdhary, Levitt, Ko, Dellit, Chesnut. Reviewed submitted version of manuscript: all authors. Approved the final version of the manuscript on behalf of all authors: Morton. Statistical analysis: Morton, Barber, Ko. Administrative/technical/material support: Barber. Study supervision: Morton, Chowdhary, Ko, Dellit, Chesnut.

\section{Correspondence}

Ryan P. Morton, Department of Neurological Surgery, University of Washington School of Medicine, 325 9th Ave., Box 359924, Seattle, WA 98104.email: rymorton@gmail.com. 